Original Article

\title{
Measurement of force sense reproduction in the knee joint: application of a new dynamometric device
}

\author{
MinooKhalkhaliZavieh,PT,PhD ${ }^{1)}$,Bahram Amirshakeri, PT $^{2}{ }^{*}$, AsGhar Rezasoltani, PT, \\ PhD $^{1)}$, Ghadam Ali Talebi, PT, PhD ${ }^{3)}$, Khosro Khademi Kalantari, PT, PhD ${ }^{1)}$, \\ Vahab Nedaey, PT, MSc ${ }^{4}$, Alireza Akbarzadeh Baghban, PhD ${ }^{1)}$ \\ 1) Department of Physical Therapy, Faculty of Rehabilitation Sciences, Shahid Beheshti University of \\ Medical Sciences, Iran \\ 2) Students Research Committee, Department of Physiotherapy, Faculty of Rehabilitation Sciences, \\ Shahid Beheshti University of Medical Sciences: Opposite to Bou-Ali Hospital, Damavand Avenue, \\ 1616913111 Tehran, Iran \\ 3) Faculty of Medicine, Babol University of Medical Science, Iran \\ 4) Physiotherapy Department, RSL Care, Queensland Health, Australia
}

\begin{abstract}
Purpose] The aim of this study was to determine the reliability of a newly designed dynamometric device for use in frequent force producing/reproducing tasks on the knee joint. [Subjects and Methods] In this cross-sectional study (Development \& Reliability), 30 young healthy males and females (age $23.4 \pm 2.48$ years) were selected among students of Tabriz University of Medical Sciences by simple randomized selection. The study instrument was designed to measure any isometric contraction force exerted by the knee joint flexor/extensor muscles, known as the ipsilateral and contralateral methods. Participant knees were fixed in $60^{\circ}$ flexion, and each participant completed the entire set of measurements twice, 72 hours apart. [Results] The findings showed a good intraclass correlation coefficient of 0.73 to 0.81 for all muscle groups. The standard error of measurement and smallest detectable difference for flexor muscle groups were 0.37 and 1.02, respectively, while the values increased to standard error of measurement $=0.38$ and smallest detectable difference $=1.05$ for extensor muscle groups. [Conclusion] The device designed could quantify the forces producing/reproducing tasks on the knee joint with a high rate of reliability, and can probably be applied for outcome measurements in proprioceptive assessment of the knee joint. Key words: Proprioception, Sense of force, Knee joint
\end{abstract}

(This article was submitted Feb. 27, 2016, and was accepted May 14, 2016)

\section{INTRODUCTION}

Proprioception plays a clear role in motor control and the postural system. Proske and Gandevia described proprioception as collective information from specialized peripheral neural endings, located at the muscle spindle, Golgi tendon, or joints, which are capable of alerting the central nervous system as to joint position, muscle force, and movement via the dorsal roots of the spinal cord ${ }^{1)}$. In addition, the sense of force, as one of the submodalities of proprioception, has also been described as tension during the contraction that plays an important role in the quality of motor function ${ }^{2}$.

Disruption in proprioception would be regarded as a risk factor to the neuromuscular system, and may result in a variety of injuries. For instance, age-related deficits in proprioception of the knee joint reportedly play an important role in initiating and advancing osteoarthritis of the knee ${ }^{3,4)}$.

*Corresponding author. Bahram Amirshakeri (E-mail: bahram_pt74@yahoo.com)

(C2016 The Society of Physical Therapy Science. Published by IPEC Inc.

This is an open-access article distributed under the terms of the Creative Commons Attribution Non-Commercial No Derivatives (by-nc-nd) License $<$ http://creativecommons.org/licenses/by-nc-nd/4.0/>. 


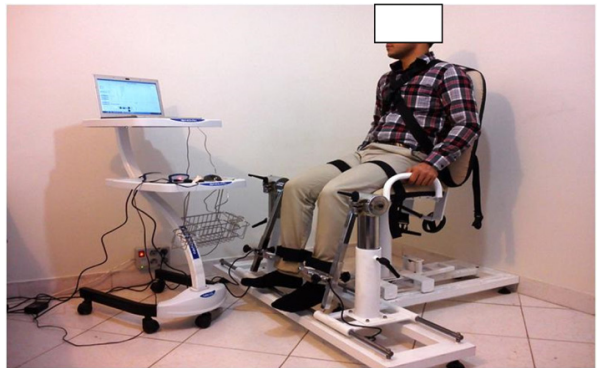

Fig. 1. Illustration of the designed device and reference position during the measurement process
Table 1. Results of one-sample t-test for standard weights, using two load cells

\begin{tabular}{ccc}
\hline \multirow{2}{*}{$\begin{array}{c}\text { Standard } \\
\text { weights }\end{array} \quad \begin{array}{c}\text { Load } \\
\text { Cells }\end{array}$} & Load cell 1 & Load cell 2 \\
\cline { 2 - 3 } $1 \mathrm{~kg}=9.8 \mathrm{~N}$ & $9.8 \pm 0.004$ & $(\mathrm{~N})$ \\
$2 \mathrm{~kg}=19.6 \mathrm{~N}$ & $19.6 \pm 0.005$ & $19.8 \pm 0.004$ \\
$3 \mathrm{~kg}=29.4 \mathrm{~N}$ & $29.3 \pm 0.005$ & $29.4 \pm 0.003$ \\
$5 \mathrm{~kg}=49 \mathrm{~N}$ & $49.0 \pm 0.04$ & $49 \pm 0.02$ \\
$10 \mathrm{~kg}=98 \mathrm{~N}$ & $98.0 \pm 0.03$ & $98 \pm 0.006$ \\
$15 \mathrm{~kg}=147 \mathrm{~N}$ & $147.0 \pm 0.04$ & $147 \pm 0.06$ \\
$20 \mathrm{~kg}=196 \mathrm{~N}$ & $196 \pm 0.06$ & $196 \pm 0.06$ \\
$25 \mathrm{~kg}=245 \mathrm{~N}$ & $245 \pm 0.007$ & $245 \pm 0.04$ \\
$30 \mathrm{~kg}=294 \mathrm{~N}$ & $294 \pm 0.007$ & $294 \pm 0.04$ \\
\hline
\end{tabular}

Data are shown as mean $\pm \mathrm{SD}, \mathrm{N}$ : Newton

Based on the literature, there are two main approaches used to measure the sense of force. The first attempts to reproduce a target force as a certain level of maximum voluntary isometric contraction (MVIC) of a muscle group in one limb or a reference side, and is known as the ipsilateral remembered method; the second aims to reproduce the same target force in the opposite limb, and is known as the contralateral method ${ }^{5-7)}$.

Evaluation of such a reproduced force sense requires a precise device with the capability of measuring muscle tension in both limbs either at the same time, or within a very short period of time. For the lower limb, the available instruments require the subjects to change their position during the force sense survey. This would interfere with the accuracy of results, as the crucial time frame allocated to each measuring test might be inadvertently exceeded. Furthermore, as the majority of knee studies have been devoted to other areas such as sense of position, pathokinesiology, kinesthesia, or compromised electromyography (EMG) patterns of knee muscles ${ }^{8-11)}$, few academic studies have addressed all major methods of force sensing measurement, including ipsilateral remembered (IR), contralateral concurrent (CC), and contralateral remembered (CR).

Because the knee is one of the joints most vulnerable to sports injuries, and due to the lack of a comprehensive measuring device for use in force sense reproducing tasks, a new device was designed, and its reliability for measuring sense of force was thoroughly tested. The need for a practical, low-cost device that can target both knee joints simultaneously or separately, with ease of use and a high rate of accuracy, unlike currently available isokinetic devices, motivated us to design such a clinically applicable instrument; this device does not require highly specialized operators or application of any invasive method to achieve results.

\section{SUBJECTS AND METHODS}

Eighty students from Tabriz University of medical sciences were included in our initial group of participants, and 54 met our inclusion/exclusion criteria. Thirty participants ( 8 female and 22 male students) were selected randomly (simple randomization) from this group of 54 eligible candidates by using RandList 1.2 software (Registration code: 1500247846). The sample size was calculated by considering $\alpha=0.05$, power $=80 \%$, and $\mathrm{r}=0.70$, with a $10 \%$ difference.

Inclusion criteria were: no history of professional sports activities, age between 20-30 years, and body mass index (BMI) between $22-25 \mathrm{~kg} / \mathrm{m}^{2}$. The exclusion criteria were: a history of knee pain and limited range of motion within the last six months, deformities, fractures, arthritis, and any systemic or neurological disorder. Subjects were informed of the principles and purpose of this study. Then, a voluntary consent form was signed by each participant to be officially considered for this study. The study protocol was approved by the Ethics Committee of Shahid Beheshti University of Medical Sciences (Tehran, Iran). The code of ethics approval number was 1391-1-94-5209, dated 5/17/2014.

The device consisted of an adjustable chair, two dynamometric mechanical arms equipped with hinged plates, two Korean manufactured load cells (Cp 500 Newton model from www.dacell.com), one USB port for intercommunication between analogue and digital components of the device using the $\mathrm{C}++$ software program (Microsoft Matlab), Math Works (a Sony laptop), VAIO Care ${ }^{\mathrm{TM}}$ Software, E Series Laptop (4-192-710-11), and connecting cables. Qt package was used for visual graphic purposes and provided visual feedback. Matlab software (Microsoft Matlab, Math Works) was utilized for trend data analysis, mean calculation, and data classification purposes (Fig. 1).

Before starting the actual tests, the accuracy of the load cells was checked by using sets of paired standard weights including 1, 2, 3, 5, 10, 15, 20, 25, and $30 \mathrm{~kg}$. Similar weights of each set were weighed by two load cells simultaneously. The results are shown in Table 1.

To minimize tactile feedback ${ }^{12}$ caused by direct contact between the lower leg and the plates, the distal portion of the lower leg was protected by a thick foam pad and a piece of thin leather used to cover the surface of each hinged leg plate.

The device chair and dynamometric mechanical arms were adjusted to support $110^{\circ}$ trunk backward extension and $60^{\circ}$ 
knee flexion, respectively. The accuracy of $60^{\circ}$ knee flexion was confirmed by a manual goniometer. Each hinged leg plate was positioned just above the middle malleolus of the corresponding leg. The plates were attached to the lower leg with Velcro straps, and excessive movement was prevented by two straps fastened around the thighs and across the trunk (Fig. 1).

During the first session, subjects became familiar with the device and study protocol. The dominant limb was determined by the subjects; the reference position was demonstrated; verbal commands and visual feedback were explained.

The study protocol included detection of maximum voluntary isometric contraction (MVIC) and two main measuring approaches known as IR and contralateral methods. The latter approach contained two subgroups known as CC and CR methods.

In order to detect MVIC, three single, strong voluntary isometric contractions, with 60 -second rest intervals, were recorded for knee flexors and extensor muscle groups separately. Each verbally prompted contraction lasted for 5 seconds, and the highest value was taken as the MVIC. Later, subjects were asked to exert only 50\% of their recorded MVIC as a target force through the producing and reproducing phases of each contraction set $\left.{ }^{4}, 12,13\right)$.

Each set of the IR method included a visually-assisted produced target force for up to 8 seconds in the reference knee joint, and a 5-second rest period, followed by a similar contraction quality of reproduced target force within the same side minus a visual cue.

The contralateral method was subdivided in two parts, i.e., CR and CC methods. While each set of the CR method consisted of a visually-assisted produced target force in the reference limb, a 5-second rest period, and a non-feedback assisted reproduced target force on the opposite side, each set of the concurrent method followed a similar protocol, but with no rest period. Both methods asked subjects to sustain their produced/reproduced target force for the entire 8 seconds ${ }^{4,12,13)}$.

One physiotherapist assessor was recruited for different data collection in each measurement session. Subjects were to alert the assessors when reaching their assumed target force, and had to sustain this to the final second. Although subjects had to complete every method in the study protocol, this process was not accomplished in any particular order, and methods could be covered randomly by each participant. Participants received 5-minutes rests between any two methods, and a 3-minute rest before initiating the next set of contractions within the same method.

To assess the test reliability in frequent measurements of force sense reproduction tasks, subjects were instructed to complete all study procedures twice, with only a 72-hour gap between test dates. In total, three strong contractions were enough to determine the MVIC in extensor and flexor muscle groups of the knee joint separately before executing 36 contractions, each equal to $50 \%$ of the MVIC. Therefore, the subjects were expected to cover 3 methods including [IR, CC, and CR] $\times 3$ sets $\times 2$ contractions $\times 2$ different muscle groups.

In order to ensure accuracy and specificity of the device, a team including a physiotherapist, designer, and biomechanical and electronic engineers met to evaluate any force sense matching error through the study period.

The accuracy of the USB port was also as high as possible, with 16-bit resolution, and a sampling rate of 64 per second. This sampling was carried out quickly and simultaneously for both limbs. The device calibration was also measured by linear regression and a one-sample t-test, as shown in Table 1.

SPSS software (Version 18.0, SPSS Inc., Chicago, IL, USA) was used for data analysis. The data distribution was evaluated using the Shapiro-Wilk test, and deemed normal after an acceptable minimum score of 0.112 was achieved. The difference between each produced and reproduced target force was recorded for any set of contractions, and considered as an absolute value to disregard the direction of the error. The average of these values for every three sets of contractions in any method (flexor/extensor) was later calculated to determine the absolute error of the mean for that method.

In order to normalize the error values as a fair percentage of the recorded MVICs for all participants, the error of the mean for each method was divided by the MVIC and the result was multiplied by 100 [The Error of the Mean/MVIC $\times 100]^{6,13,14)}$.

The values of the Intraclass Correlation Coefficient (ICC) and actual units of standard error of measurement or $\mathrm{SEM}=\mathrm{SD} \times \sqrt{1-\mathrm{ICC}}$, shown in Table 3, indicate the relative absolute reliability of this study. To determine the actual changes in force reproducing error, the smallest detectable difference was calculated as $\mathrm{SDD}=\mathrm{SEM} \times 1.69 \times \sqrt{2}{ }^{15}$ ).

A paired samples t-test was conducted to compare the mean variables obtained from different dates of measurement, and Spearman's correlation coefficient test was used to investigate the existing correlation.

\section{RESULTS}

Demographic characteristics of participants and MVIC mean value of flexor and extensor muscle groups are shown in Table 2 .

In evaluating the calibration with a linear regression test, $R$ Square $=1$ and R Square $=0.999$ were used for load cells one and two, respectively.

The ICC ranged from 0.73 to 0.81 . The highest confidence rates were attributed to methods $C C=0.81$ and $C R$, and $C C=0.80$ for knee flexor and extensor muscle groups, respectively (Table 3).

The pooled data did not show a statistically significant difference between the first and the second date of the study (paired sample t-test and Spearman's correlation coefficient test). However, the average value of mean \pm SD and SEM for the CR method was higher for both muscle groups in this experiment. The least value for SDD was attributed to the CC method in the flexor muscle group, at 1.02, while the CR method in the flexor muscle groups had the highest SDD rate, at 2.04. 
Table 2. Demographic characteristics and mean maximum voluntary isometric contraction

\begin{tabular}{lcc}
\hline Variable & Variable levels & Mean $\pm \mathrm{SD}$ \\
\hline Age (years) & & $23.4 \pm 2.5$ \\
BMI $\left(\mathrm{kg} / \mathrm{m}^{2}\right)$ & & $2.1 \pm 9.22$ \\
MVIC & Day 1 & $173.3 \pm 33.7 \mathrm{n}$ \\
(knee flexors) & Day 2 & $167.8 \pm 25.4 \mathrm{n}$ \\
MVIC & Day 1 & $336.2 \pm 73 \mathrm{n}$ \\
(knee extensors) & Day 2 & $348.7 \pm 65 \mathrm{n}$ \\
\hline \multicolumn{2}{l}{ MVIC: maximum voluntary isometric contraction; $\mathrm{n}$ : newton }
\end{tabular}

Table 3. The mean standard deviation, interclass correlation coefficient (ICC), standard error of measurement (SEM), and smallest detectable difference (SDD) for three force sense measuring methods of knee flexor and extensor muscle groups

\begin{tabular}{|c|c|c|c|c|c|c|}
\hline \multirow[t]{2}{*}{ Variable } & Measuring & First session & Second session & \multirow[t]{2}{*}{ ICC } & \multirow[t]{2}{*}{ SEM } & \multirow[t]{2}{*}{ SDD } \\
\hline & methods & \multicolumn{2}{|c|}{ Mean \pm SD } & & & \\
\hline \multirow{3}{*}{$\begin{array}{l}\text { Extensor } \\
\text { muscles }\end{array}$} & IR & $2.06 \pm 0.89$ & $0.81 \pm 1.92$ & 0.76 & 0.38 & 1.05 \\
\hline & $\mathrm{CC}$ & $2.10 \pm 0.90$ & $2.20 \pm 0.99$ & 0.8 & 0.48 & 1.32 \\
\hline & $\mathrm{CR}$ & $2.97 \pm 1.41$ & $3.13 \pm 1.52$ & 0.8 & 0.71 & 1.96 \\
\hline \multirow{3}{*}{$\begin{array}{l}\text { Flexor } \\
\text { muscles }\end{array}$} & IR & $2.50 \pm 1.21$ & $2.76 \pm 1.24$ & 0.73 & 0.45 & 1.24 \\
\hline & $\mathrm{CC}$ & $3.01 \pm 1.38$ & $2.83 \pm 1.34$ & 0.81 & 0.37 & 1.02 \\
\hline & $\mathrm{CR}$ & $3.15 \pm 1.46$ & $3.20 \pm 1.56$ & 0.73 & 0.74 & 2.04 \\
\hline
\end{tabular}

IR: ipsilateral remembered, CC: contralateral concurrent, CR: contralateral remembered

\section{DISCUSSION}

Absence of meaningful differences across values of mean \pm SD for the reproduced target force $(\mathrm{p}<0.05)$ were convincing enough; that no systemic bias could interfere with the results. The device reliability for assessing force sense reproduction across the different study test methods, 0.73 to 0.81 , was consistent with what Munro previously described as a high class of reliability ${ }^{16)}$. In this classification, ICC ranges from 0 to 0.25 and 0.26 to 0.49 are known as poor and weak, respectively, while a moderate ICC must show a range of 0.50 to 0.69 . Within this classification, the high and very high ranges of reliability are identified as 0.70 to 0.89 and 0.90 to 0.100 , respectively ${ }^{16)}$.

In order to evaluate the device reliability in this study, and for measurement of the force sense reproduction tasks, the mean \pm SD was calculated in a manner similar to that used by Docherty, Dover, and their associates throughout their force sense experiments $7,12,17)$

When errors associated with frequent measurements are considered, SEM will assist in determining the range within which the subject's true score is expected to lie $^{18)}$. This provides the value of measurement error in the same units as the measurement itself. SEM is also more applicable for daily clinical decision-making than ICC itself, which can sometimes be difficult to interpret ${ }^{15,19)}$. In fact, SEM is inversely correlated with absolute reliability, and thus the accuracy of the obtained measurements ${ }^{20,21)}$. In this study, the SEM recorded through a sequence of frequent measuring methods in reproducing the target force was relatively small, at 0.37 to 0.74 . SDD, indicating the smallest statistically significant change in measurement results, would enable us to estimate the actual error in force reproducing tasks. In fact, the smaller range of SDD would allow those true changes in different tests to be detected more precisely, or those larger than the defined SDD to be considered as true errors in force reproducing methods ${ }^{15}$.

In general, regulating muscle tension and the sense of force should vary among individuals. The variations in those who perform precise work, such as musicians, are much smaller, whereas the reverse is true for those who perform powerful, coarse tasks ${ }^{22}$.

The term, just-noticeable difference (JND), quantifies how different two stimuli must be before subjects reliably report them as distinct ${ }^{23)}$. A $5-10 \%$ JND was previously reported by Weber for producing and controlling muscle force when participants were asked to hold a 975 -g weight in their hands ${ }^{24,25)}$. Thus, some noticeable differences in measurement of force sense reproducing tasks through two study dates could be attributed to variations in this sense rather than accuracy of the device. However, as no specific research has measured the accuracy of force sense reproducing tasks of the knee joint based on this study, no direct comparison can be determined between our findings and previous studies. 
Based on the results of this study, all test methods demonstrated acceptably high reliability with a small standard error in frequent measuring tasks. Therefore, for measurement of force sense reproduction in knee joint flexor/extensor muscle groups, this device can be applied for diagnostic and therapeutic procedures with a great degree of reliability.

The cost-effectiveness of this device in comparison with commercial isokinetic devices, and its high precision to a level of one-hundredth of a Newton, along with its ease of use and comfort for operator and subjects, are major advantages. Added advantages would include simultaneous bilateral force sense evaluations on the lower limbs, which are equally applicable to upper limb measurements with a small adjustment in the dynamometric arms of the device. The device offers no health or safety risks to operators or subjects.

The device was only able to measure the sense of force and reproduced force through isometric contractions, and the sample was exclusively composed of normal subjects.

\section{REFERENCES}

1) Proske U, Gandevia SC: The proprioceptive senses: their roles in signaling body shape, body position and movement, and muscle force. Physiol Rev, 2012, 92 : 1651-1697. [Medline] [CrossRef]

2) Henningsen H, Knecht S, Ende-Henningsen B: Influence of afferent feedback on isometric fine force resolution in humans. Exp Brain Res, 1997, 113: 207-213. [Medline] [CrossRef]

3) Segal NA, Glass NA, Felson DT, et al.: Effect of quadriceps strength and proprioception on risk for knee osteoarthritis. Med Sci Sports Exerc, 2010, 42: 2081-2088. [Medline] [CrossRef]

4) Adamo DE, Martin BJ, Brown SH: Age-related differences in upper limb proprioceptive acuity. Percept Mot Skills, 2007, 104: 1297-1309. [Medline]

5) Kim CY, Choi JD, Kim HD: No correlation between joint position sense and force sense for measuring ankle proprioception in subjects with healthy and functional ankle instability. Clin Biomech (Bristol, Avon), 2014, 29: 977-983. [Medline] [CrossRef]

6) Scotland S, Adamo DE, Martin BJ: Sense of effort revisited: relative contributions of sensory feedback and efferent copy. Neurosci Lett, $2014,561: 208-212$. [Medline] [CrossRef]

7) Docherty CL, Arnold BL, Hurwitz S: Contralateral force sense deficits are related to the presence of functional ankle instability. J Orthop Res, 2006, 24: 1412-1419. [Medline] [CrossRef]

8) de Vries AJ, van den Akker-Scheek I, Diercks RL, et al.: The effect of a patellar strap on knee joint proprioception in healthy participants and athletes with patellar tendinopathy. J Sci Med Sport, 2016, 19: 278-282. [Medline] [CrossRef]

9) Ma Y, Deie M, Iwaki D, et al.: Balance ability and proprioception after single-bundle, single-bundle augmentation, and double-bundle ACL reconstruction. ScientificWorldJournal, 2014, 2014: 342012. [Medline] [CrossRef]

10) Bouchouras G, Patsika G, Hatzitaki V, et al.: Kinematics and knee muscle activation during sit-to-stand movement in women with knee osteoarthritis. Clin Biomech (Bristol, Avon), 2015, 30: 599-607. [Medline] [CrossRef]

11) Schroeder MJ, Krishnan C, Dhaher YY: The influence of task complexity on knee joint kinetics following ACL reconstruction. Clin Biomech (Bristol, Avon), 2015, 30: 852-859. [Medline] [CrossRef]

12) Dover G, Powers ME: Reliability of joint position sense and force-reproduction measures during internal and external rotation of the shoulder. J Athl Train, 2003, 38: 304-310. [Medline]

13) Adamo DE, Scotland S, Martin BJ: Asymmetry in grasp force matching and sense of effort. Exp Brain Res, 2012, 217: 273-285. [Medline] [CrossRef]

14) Kues JM, Rothstein JM, Lamb RL: Obtaining reliable measurements of knee extensor torque produced during maximal voluntary contractions: an experimental investigation. Phys Ther, 1992, 72: 492-501, discussion 501-504. [Medline]

15) Donoghue D, Stokes EK, Physiotherapy Research and Older People (PROP) group: How much change is true change? The minimum detectable change of the Berg Balance Scale in elderly people. J Rehabil Med, 2009, 41: 343-346. [Medline] [CrossRef]

16) Munro BH: Statistical methods for health care research, 5th ed. Philadelfia: JB Lippincott Williams and Wilkins, 2005.

17) Smith BI, Docherty CL, Simon J, et al.: Ankle strength and force sense after a progressive, 6-week strength-training program in people with functional ankle instability. J Athl Train, 2012, 47: 282-288. [Medline]

18) Ross M: Test-retest reliability of the lateral step-up test in young adult healthy subjects. J Orthop Sports Phys Ther, 1997, 25: 128-132. [Medline] [CrossRef]

19) Weir JP: Quantifying test-retest reliability using the intraclass correlation coefficient and the SEM. J Strength Cond Res, 2005, 19: 231-240. [Medline]

20) Talebi GA, Oskouei AE, Shakori SK: Reliability of upper limb tension test 1 in normal subjects and patients with carpal tunnel syndrome. J Back Musculoskeletal Rehabil, 2012, 25: 209-214. [Medline]

21) Mathur S, Eng JJ, MacIntyre DL: Reliability of surface EMG during sustained contractions of the quadriceps. J Electromyogr Kinesiol, 2005, 15: 102-110. [Medline] [CrossRef]

22) Semmler JG, Nordstrom MA: Motor unit discharge and force tremor in skill- and strength-trained individuals. Exp Brain Res, 1998, 119: 27-38. [Medline] [CrossRef]

23) Acuna DE, Berniker M, Fernandes HL, et al.: Using psychophysics to ask if the brain samples or maximizes. J Vis, 2015, 15: 15. [Medline] [CrossRef]

24) Prasad MS, Prswani S, Manivannan M: Force JND for right index finger using contra lateral force matching paradigm. Lecture Notes in Mech Eng, $2013,13$. [CrossRef]

25) Cody FW, Garside RA, Lloyd D, et al.: Tactile spatial acuity varies with site and axis in the human upper limb. Neurosci Lett, 2008, 433: 103-108. [Medline] [CrossRef] 